$00216 \mathrm{~A}$

\title{
SURFACE AMINATION OF POLY(ACRYLONITRILE)
}

\author{
ANDREAS HARTWIG, MARCEL MULDER* and CEES A. SMOLDERS \\ University of Twente, Faculty of Chemical Technology, P.O. Box 217, \\ 7500 AE Enschede, The Netherlands
}

\section{CONTENTS}

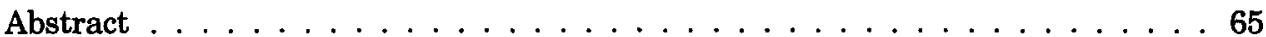

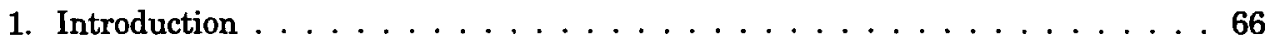

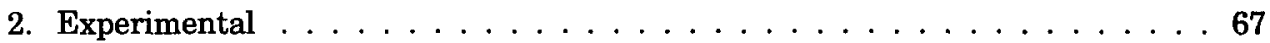

2.1 Plasma Treatment . . . . . . . . . . . . . . . . . . . . . . . . . . .

2.2 Film Preparation . . . . . . . . . . . . . . . . . 68

2.3 Determination of Amino Groups . . . . . . . . . . . . . . 68

3. Results and Discussion . . . . . . . . . . . . . . . . 69

3.1 Ammonia Plasma Treatment . . . . . . . . . . . . . . . 69

3.1.1 Influence of the Discharge Time . . . . . . . . . . . . . . 70

3.1.2 Variation of the Pressure . . . . . . . . . . . . . . 70

3.1.3 Variation of the Plasma Power . . . . . . . . . . . . . . 71

3.2 Decomposition of Amino Group Containing Chemicals on the Surface

by Noble Gas Plasmas . . . . . . . . . . . . . . . . . . . . 76

3.3 Reduction of the Nitrile Groups . . . . . . . . . . . . . . . 76

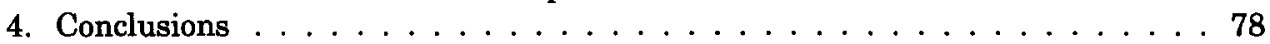

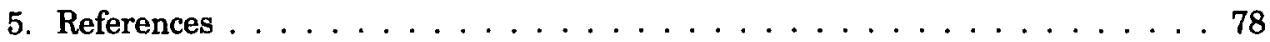

\section{ABSTRACT}

The surface amination of poly(acrylonitrile) by ammonia plasma treatment has been studied. Furthermore, two other surface modification techniques have been investigated, the plasma chemical decomposition of an amino group containing chemical (tris-(2-aminoethyl)amine) onto the polymer surface and the surface reduction by lithium aluminium hydride. The three different methods are compared with respect to the adhesion improvement of the coatings onto the modified surfaces.

The number of groups introduced on the surfaces has been determined by a wet chemical method.

* Corresponding author. 


\section{INTRODUCTION}

The surface modification of polymers is an area of considerable technological and academic importance. The surface treatment methods can be classified into two general categories depending whether a wet chemical process is involved or whether the interaction occurs at a gas-solid interface as in plasma discharge treatment. The surface properties are important with respect to the wettability or the printing and coating behaviour of the polymers. It is also possible to immobilize enzymes on the modified surfaces [1]. A strong interaction between support and coating polymer is also necessary in the preparation of composite membranes.

Dense polymer membranes are only applicable when their permeate flux is high enough. In order to achieve this, the effective layer should be as thin as possible. However, very thin polymer layers are mechanically unstable without a support layer, which means that a porous support is necessary. Many substrates are available and for this study porous poly(acrylonitrile) (PAN) has been used. A stable substrate/coating-laminate can be obtained when both layers have a strong mutual interaction. It is well known that the adhesion can be enhanced by functionalizing the support surface by plasma discharges. Because of the lower degree of degradation in the main chain, ammonia plasmas are preferred over oxygen plasmas. For example, Chappell [2] showed that the adhesion of epoxy and unsaturated polyester resins on poly(ethylene) is enhanced after ammonia plasma treatment of the poly(ethylene). Many membrane polymers contain carboxylate- and ester-groups, such as the resins used by Chappell, which can interact with specific basic groups, i.e. aminogroups, formed by an ammonia plasma. Therefore, this work has been focused on surface modification by ammonia plasmas.

If the adhesion of the coating on the substrate is too low even after the plasma treatment, the combination of amino-groups on the substrate and acidic end groups of the membrane polymer allows a chemical coating on the substrate. If the formation of this chemical bond is too slow or if the given functional groups of the coating polymer are not reacting with the surface amino groups, a further functionalization is possible. Urea bonds can be formed by the reaction of the surface amino groups with a diisocyanate. The second isocyanate group of this diisocyanate is available for reactions with a wide variety of coating polymer functionalities. 
The formation of amino groups on PAN surfaces is not only possible by an ammonia plasma treatment. Also the chemical reduction of the PAN nitrile groups by lithium aluminium hydride [3], which results in primary amino groups only, is possible. Therefore, PAN is a very suitable polymer to compare the wet chemical and plasma chemical formation of amino groups on the surface.

Various analytical methods are available to obtain detailed information about the number of amino groups formed on the surface. A spectroscopic method to analyse surfaces (e.g. ESCA, ATR-FTIR) does not only reveal the state of the top surface, which is accessible for chemical interactions and reactions, but also the state of the first couple of atomic layers underneath. A chemical method does not have this disadvantage, and only the groups which are accessible for a chemical reaction, are detected. Because of the difficulty to analyse the surface of porous materials, the modification of the PAN surface, as reported here, should be performed with nonporous PAN films. These results must then can be related to porous PAN membranes.

It is assumed that the surface composition of the polymer and of the bulk material are equal, that the atoms are balls and the surface is flat. Now, it is possible to estimate the number of monomer units per square nanometer. With a density of $1.17 \mathrm{~g} / \mathrm{cm}^{3}$ and a molecular mass of the monomer of $53 \mathrm{~g} / \mathrm{mol}$, one may calculate 4.6 monomer units per square nanometer PAN film. The number of formed functional groups on the surface should be in this range, but since polymer surfaces are never flat in the molecular range, this is only a very rough estimation.

\section{EXPERIMENTAL}

\subsection{Plasma Treatment}

An Electrotec "Plasmafab PF 508" plasmareactor was used for the plasma modification of the polymer films. The radio-frequency employed was $13.56 \mathrm{MHz}$, the volume of the chamber 36 liter. The pressure was measured by a Pirani gauge and adjusted by a hand driven valve in the pumping line. In later experiments the pressure control was performed automatically by an absolute pressure gauge (Baratron) and a motor driven throttle valve. Gas fluxes were controlled by a mass flow controller (Brooks). 
The polymer samples (usually samples of 26 by $76 \mathrm{~mm}$ ) were fixed with tape on a glass plate, which was placed on the Faraday-cage electrode of the reactor. To ensure a stable surface state, the samples were usually stored in air after plasma treatment for one day before they were analysed or treated further. The experimental conditions of the treatment are given below.

\subsection{Film Preparation}

A $0.15 \mathrm{~mm}$ thick layer of a $15 \%$ solution (w/w) of poly(acrylo nitrile) (Bayer; T-Pulver) in dimethyl formamide was casted on a glass plate. The solvent was evaporated overnight in a nitrogen atmosphere. Some of the films were annealed at $80^{\circ} \mathrm{C}$ for $14 \mathrm{~h}$. The resulting films are approximately $13 \mu \mathrm{m}$ thick and the most intensive infrared absorptions are in the range of $10 \%$. Because the absorptions in the spectra of these films never reach $0 \%$, subtraction of spectra may reveal small composition changes. Some experiments are carried out with unannealed films, but this will be mentioned in the text.

\subsection{Determination of Amino Groups}

Methyl orange is an acid dye which binds with amino group containing substrates, and the dye can be removed again by bases [4]. This behaviour can be used to determine the number of amino groups on polymer surfaces.

The modified films were treated with the acidic methyl orange solution (0.05\% methyl orange in a $0.1 \mathrm{~m}$ solution of sodium dihydrogen phosphate in water, filtered; $\mathrm{pH}$ app. 4.7) for $1 \mathrm{~h}$. After rinsing the excess of dye with water the samples were carefully pressed between tissue paper to remove the water. The fixed methyl orange was resolved by $5 \mathrm{ml}$ of a $0.1 \mathrm{~m}$ potassium carbonate solution and the concentration determined photometrically at $465 \mathrm{~nm}$. A $1 \mathrm{~cm}$ cell was used with a pure potassium carbonate solution as reference. At the chosen conditions the molar extinction coefficient of the methyl orange is $\varepsilon=-23250 \mathrm{l} /(\mathrm{mol} \mathrm{cm})$. The number of amino groups $C^{\prime}\left[1 / \mathrm{nm}^{2}\right]$ on the surface can be determined with this constant, the surface $\mathrm{F}\left[\mathrm{cm}^{2}\right]$ and the actual measured absorption Abs:

$\mathrm{C}^{\prime}=1300 \cdot \mathrm{Abs} / \mathrm{F}$ 
The main disadvantage of this method is that the number of detectable groups is restricted to the maximum packing density of the methyl orange molecules at the surface.

\section{RESULTS AND DISCUSSION}

\subsection{Ammonia Plasma Treatment}

Ammonia becomes fragmented in a glow discharge. The fragments are determined by the power input per molecule; they can vary from $\mathrm{NH}_{2}$ radicals to nitrogen and hydrogen. There is always a distribution of different fragments, which affects the resulting surface composition. The formation of stable new gases is clear from the pressure increase after generating the plasma. This increase is much more distinct than with plasma gases that cannot form stable products in the plasma, e.g. oxygen.

To avoid problems in reproducibility of a PAN film, the points in a certain figure are always obtained from one PAN film.

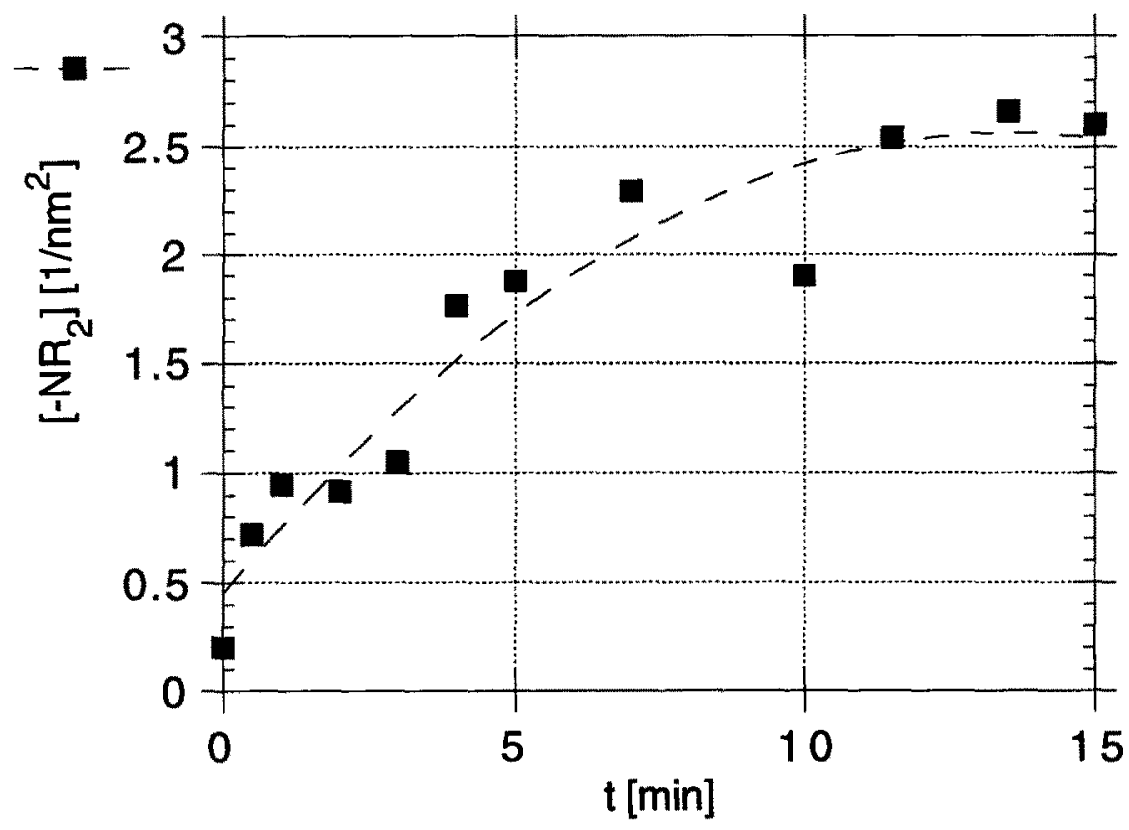

Fig. 1. Number of basic groups formed on the PAN surface after different times of treatment with an ammonia plasma. 


\subsubsection{Influence of the Discharge Time}

To exclude influences of time in the examination of the other plasma parameters with respect to the surface composition, one must be sure that the surface composition remains constant after the chosen discharge time. Figure 1 shows the number of basic groups after treatment times between 0 and $15 \mathrm{~min}$. The other plasma parameters were; $P_{n}=100 \mathrm{~W}$, $\Phi\left(\mathrm{NH}_{3}\right)=58 \mathrm{~cm}^{3}(\mathrm{STP}) / \mathrm{min}$ and $\mathrm{p}=0.2 \mathrm{mbar}$. The number of basic groups increases with increasing treatment times up to 10 minutes and becomes constant at longer times. A treatment time of 15 minutes was chosen for further experiments to eliminate time effects.

\subsubsection{Variation of the Pressure}

The pressure was varied between 0.2 and $1.1 \mathrm{mbar}$ to investigate whether this parameter has an influence on the number of introduced basic groups onto the PAN surface. Other parameters were: $P_{n}=100 \mathrm{~W}$, $\Phi\left(\mathrm{NH}_{3}\right)=58 \mathrm{~cm}^{3}(\mathrm{STP}) / \mathrm{min}$ and $\mathrm{t}=15 \mathrm{~min}$. The results are given in Fig. 2. At low pressures the number of formed basic groups is strongly decreasing with increasing pressure. Obviously the energy feed into the reactor is better dissipated. This energy dissipation leads to more, but less reactive excited particles. From a pressure of 0.7 mbar a further

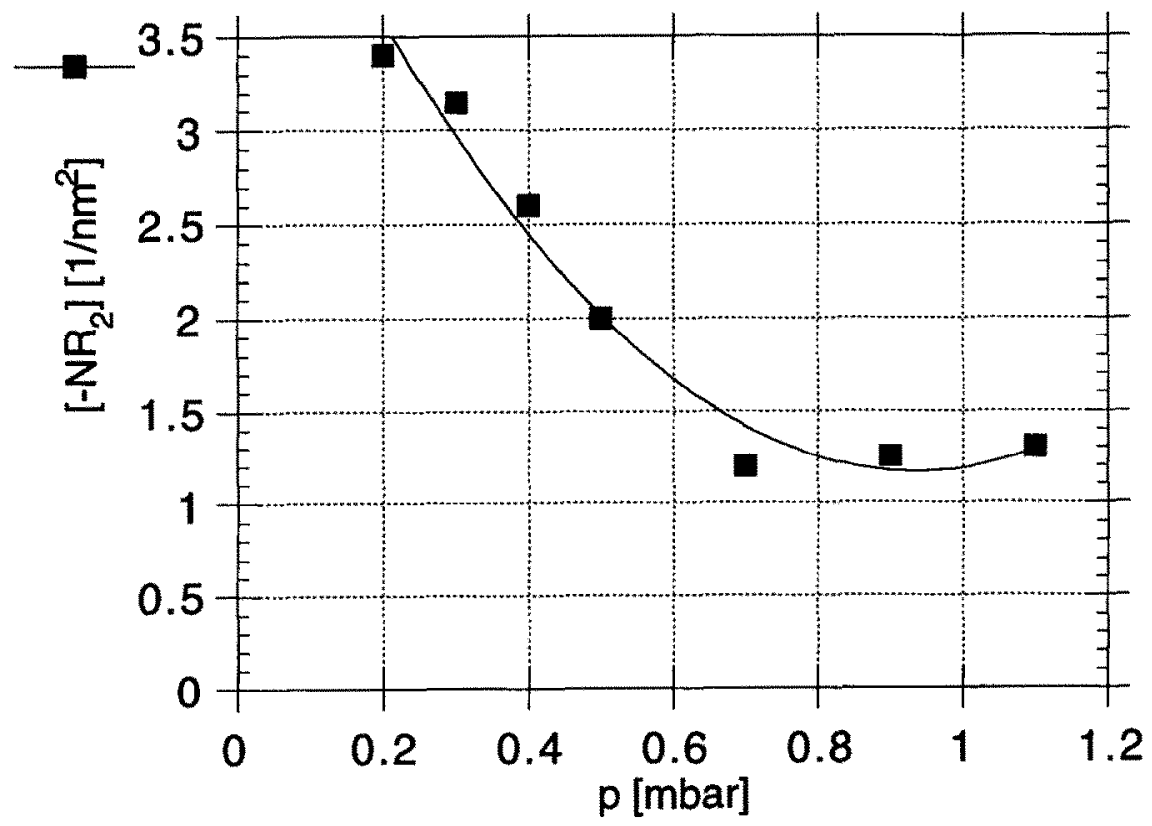

Fig. 2. Number of amino groups formed by ammonia plasmas at various pressures. 
dissipation does not take place. All the excited particles are in the lowest excitation state, which implies that the energy state of the reactive particles remains constant with the pressure. This is demonstrated by the constant number of formed amino groups on the surface of samples treated with plasmas of pressures above $0.7 \mathrm{mbar}$. Since the number of basic groups is the highest at the lowest possible pressure ( $0.2 \mathrm{mbar})$ with the chosen gas flow, all further experiments were performed at this pressure.

\subsubsection{Variation of the Plasma Power}

Figure 3 shows the influence of the plasma power on the number of basic groups formed onto the PAN surface. The other plasma parameters were; $\mathrm{t}=15 \mathrm{~min}, \mathrm{p}=0.2 \mathrm{mbar}$ and $\Phi\left(\mathrm{NH}_{3}\right)=58 \mathrm{~cm}^{3}(\mathrm{STP}) / \mathrm{min}$. The influence of the plasma power on the surface composition of poly(propylene) (PP), which was treated under the same conditions, is shown in Fig. 3 as well. It is not possible to compare the absolute numbers of basic

$-\wedge-P P$

- - - PAN

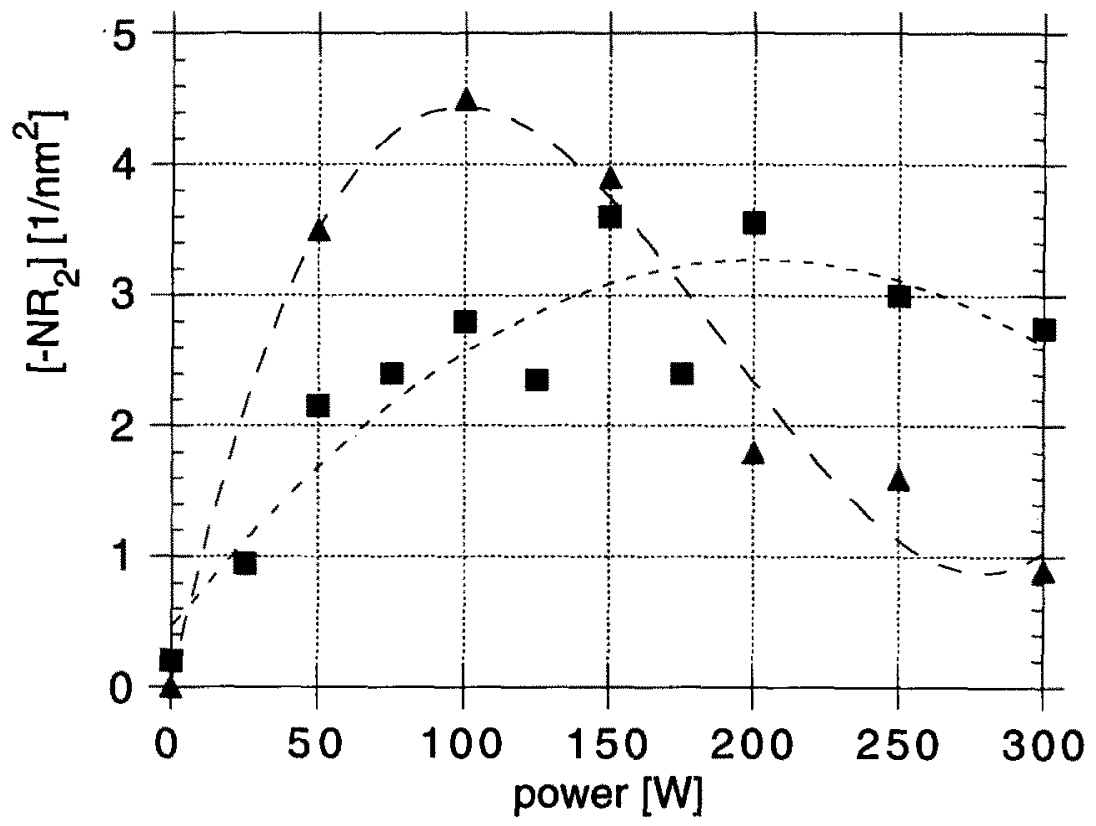

Fig. 3. Number of basic groups formed on PAN and poly(propylene) after treatment with ammonia plasmas of different powers. 
groups found for the two different polymers, because the results refer to the macrosurface; the actual microsurface is unknown. But it is interesting to compare the trends of the two graphs. The graph for PP goes through a very distinct maximum, whereas the number of basic groups on PAN remains nearly constant at high powers. This result shows, that at high powers a large content of the ammonia becomes decomposed and the small fragments, such as $\mathrm{H}, \mathrm{H}_{2}$ or $\mathrm{NH}$, are not any longer able to form directly basic groups. The nitrile groups of the PAN can however be reduced by the hydrogen formed in the plasma. This leads not exclusively to primary amino groups, since the modified surface is changed by collisions of plasma particles. This mechanism of basic group formation does not hold for PP and this will result in a decreasing number of basic groups with increasing power.

Contact angle measurements may give information about hydrophilicity of the modified surface and whether more hydrophilic (primary) or more hydrophobic (tertiary) amino groups are present. Contact angle measurements were performed with $1 \mu l$ drops of triple distilled water. The found results are shown in Fig. 4.

The decreasing contact angle shows that at powers below $75 \mathrm{~W}$ the hydrophilicity of the surface increases. With a further increase in power the hydrophilicity of the surface decreases again. Samples treated with a power of $300 \mathrm{~W}$ show nearly the same contact angle as unmodified PAN. At low powers the power input is not high enough to form a sufficient number of amino groups, as shown above (see Fig. 3), but mostly primary amino groups with a highly hydrophilic character are being formed. When the power increases the ammonia becomes more readily decomposed, forming less hydrophilic secondary and tertiary amino groups, and amides. A treatment power of $100 \mathrm{~W}$ seems to be a good compromise to form surfaces with a high density of desired primary amino groups.

The samples were stored in air for either 4 days or 20 days before the contact angles were measured. The difference between the graphs shows that even after 4 days the surface composition is not constant: most likely the surface adsorbs water from the air, which increases the mobility of the surface groups, so that they can turn away from the surface. The smaller difference between the two curves with larger power shows that the surface mobility becomes lower at high powers, caused by the lower hydrophilicity and the higher degree of crosslinking. The shown results were obtained with PAN films annealed for $14 \mathrm{~h}$ at $80^{\circ} \mathrm{C}$ prior to plasma treatment. Nevertheless, also experiments with unannealed PAN sam- 


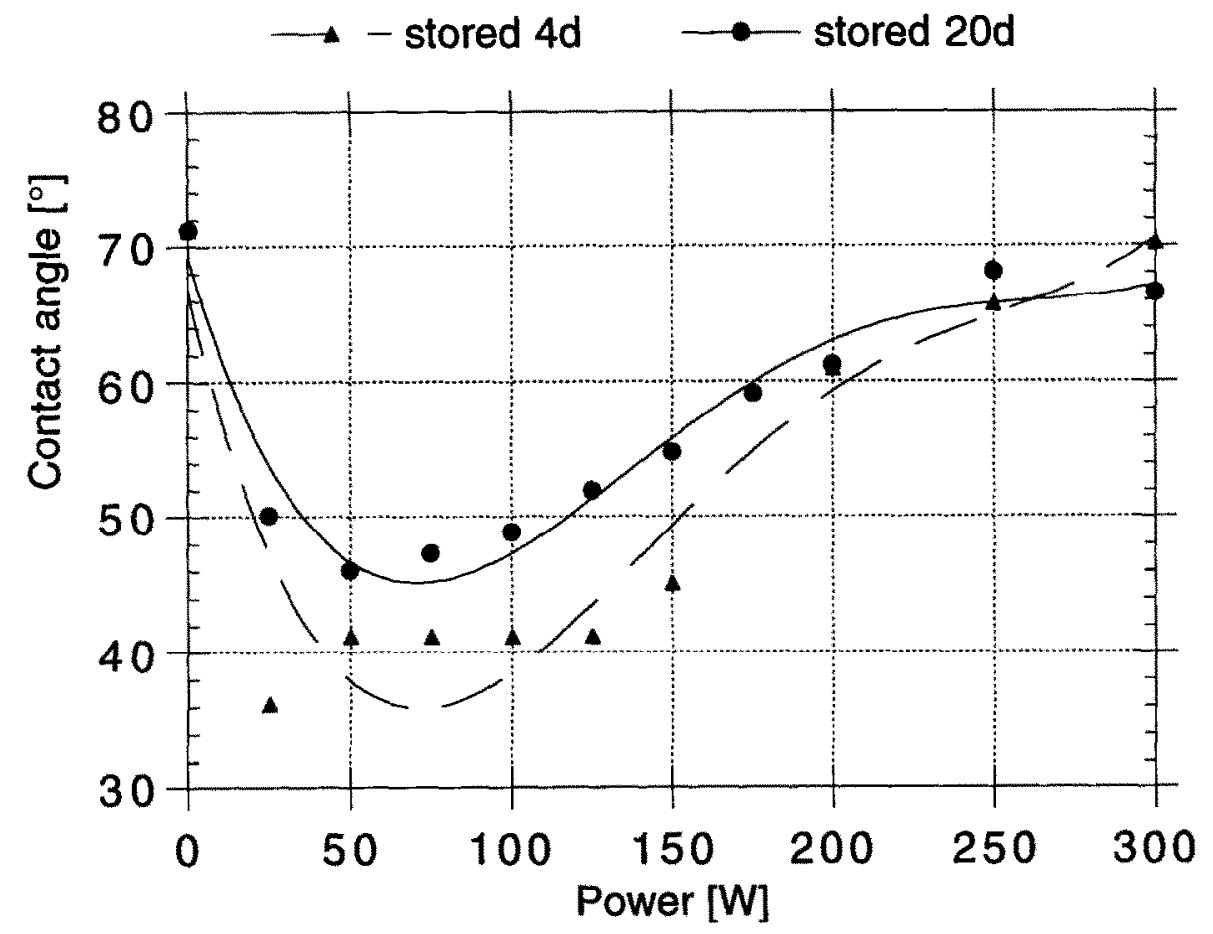

Fig. 4. Contact angles of water on PAN treated with ammonia plasmas of different powers after storing in air for either 4 days (4d) or 20 days (20d).

ples were performed. Figure 5 shows the number of basic groups found on samples treated with ammonia plasmas of different powers. The samples were analysed twice, and surprisingly a large difference can be found between the two series. The first analysis shows a significantly higher number of basic groups compared to the second one. The results obtained in the second analysis are fairly the same as found for the annealed samples, which did not show a difference between the first and second analysis.

Obviously the treatment of the modified PAN film with the analytical solutions leads to a similar effect as annealing of the films. If the first (acidic) solution would have an influence on the number of observed basic groups this would have an effect on the first dye analysis and no difference should be found between the two analyses. Consequently the second (basic) solution must cause the difference. Since basic groups are present on the surface, a more hydrophilic character is obtained which will exhibit a higher degree of swelling in aqueous solutions. It is known [5], that PAN films casted from solutions have a rough surface, which is 


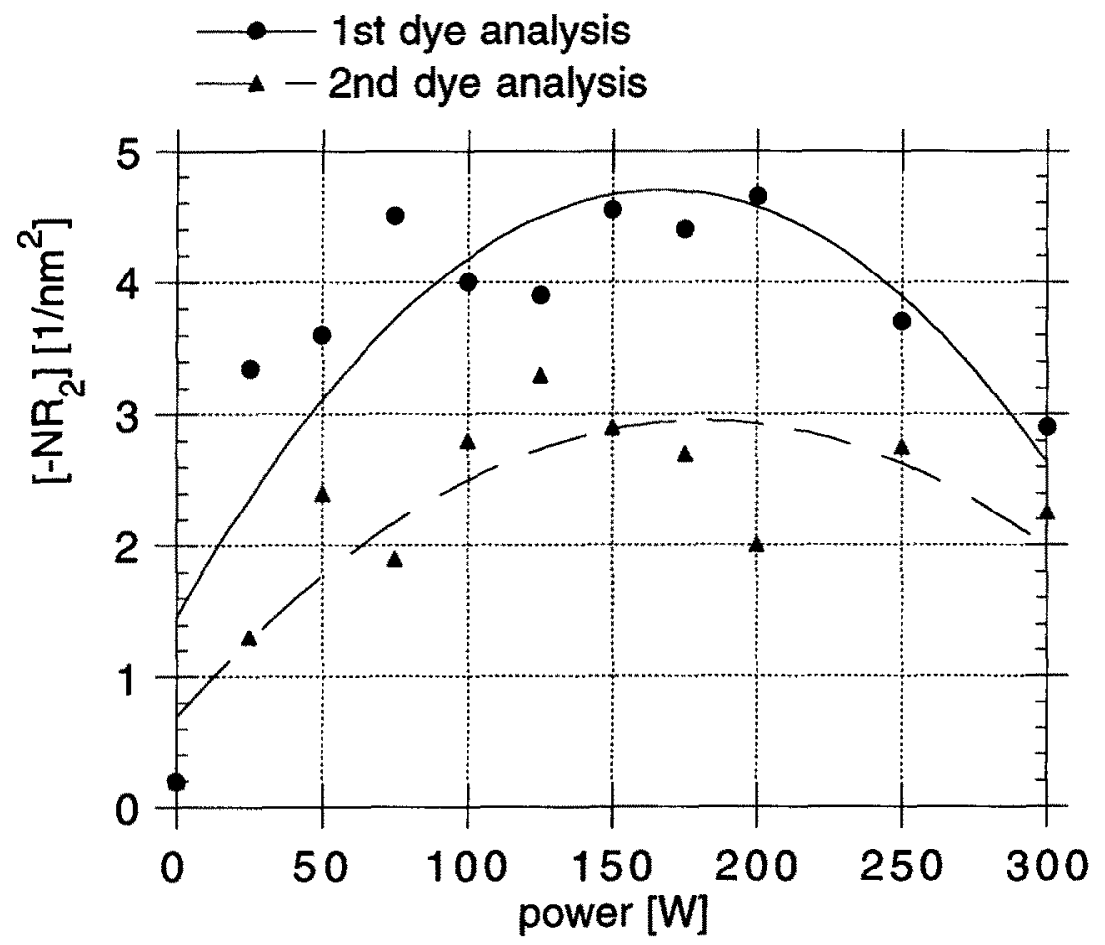

Fig. 5. Number of basic groups from the first and second dye analysis on unannealed PAN films after treatment with ammonia plasmas of different powers.

caused by the polymer crystallinity. From an energetic point of view the surface should be as small as possible. Swelling of the surface in the basic analytical solution increases the chain mobility in the same way as it does during annealing. Therefore, both treatments promote the formation of a thermodynamically more stable state with a lower interface energy, resulting in a decreased surface roughness.

To support this model atomic force microscopic studies were performed. Samples were measured with a constant force between tip and surface as well as with a constant distance. Figure 6 shows pictures taken with a constant force. It can be observed that the surface roughness is less for the annealed sample (average roughness $\approx 3.1 \mathrm{~nm}$ ) and the sample

Fig. 6. Surface structures from atomic force microscopy (AFM) of different PAN samples. (a) Film casted from DMF solution (15\%). (b) Sample (a) treated for 15 min with a $100 \mathrm{~W}$ ammonia plasma and a $0.1 \mathrm{~m} \mathrm{~K}_{2} \mathrm{CO}_{3}$ solution. (c) Sample (a) annealed for $14 \mathrm{~h}$ at $80^{\circ} \mathrm{C}$. 
(a)

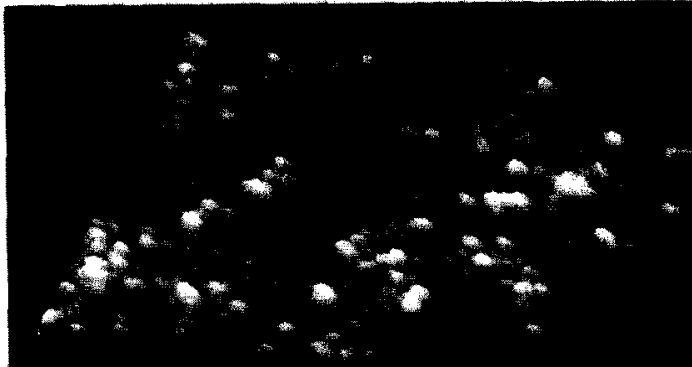

(b)

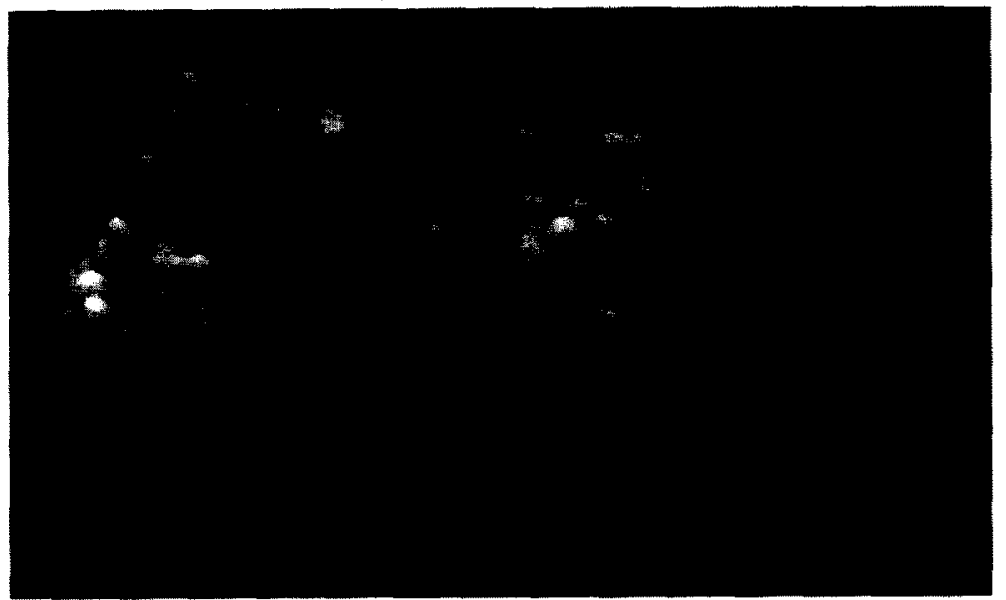

(c)

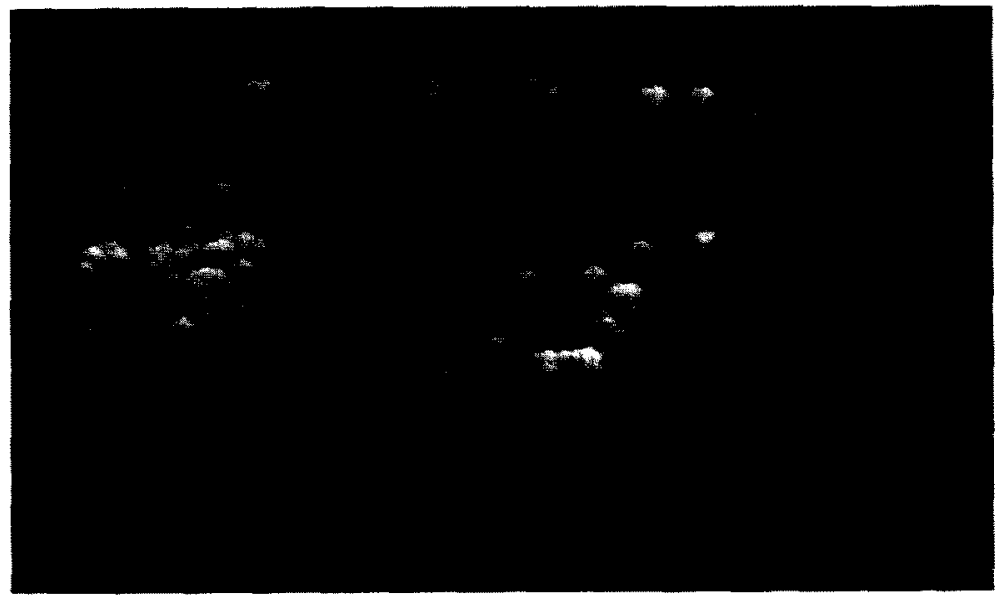

Fig. 6. Caption opposite. 
treated with ammonia plasma and $0.1 \mathrm{~m}$ potassium carbonate solution (average roughness $\approx 3.3 \mathrm{~nm}$ ) compared to the untreated film (average roughness $\approx 4.0 \mathrm{~nm}$ ). The flatter surface structures lead to a lower effective surface, resulting in a lower number of amino groups.

\subsection{Decomposition of Amino Group Containing Chemicals on the Surface by Noble Gas Plasmas}

It should be possible to fix amino groups at the surface of a polymer by reaction of an amino group containing compound onto the polymer surface. The plasma forms radicals by decomposition of the polymer surface and the compounds at the surface and these radicals can then combine. Tris (2-aminoethyl)amine $\left(\mathrm{N}_{2}\left(\mathrm{C}_{2} \mathrm{H}_{4} \mathrm{NH}_{2}\right)_{3}\right)$ was chosen as an amino group containing compound. The amino group content is very high with three primary amino groups per molecule. In addition, this compound has a bigh boiling point $\left(114^{\circ} \mathrm{C} / 15\right.$ Torr $)$, and it will remain on the polymer surface during treatment in vacuum. Since it is an oily substance it is easy to coat on the polymer surface. Helium was chosen as plasma gas to decompose the tris(2-aminoethyl)amine. This gas has the highest ionization potential $(24.5 \mathrm{eV})$ which ensures a maximum depth of modification and degree of fragmentation. The PAN films were put into the tris(2-aminoethyl)amine for 14 hours, the excess of amine was removed from the surface, whereafter the samples were treated with the helium plasma. During this experiment, a white solid layer was formed at the PAN surface. After washing this layer with water from the PAN surface, the samples were analysed as described above. The number of basic groups formed with this method was over a large range of powers and treatment times always lower than with the ammonia plasma and the reproducibility was very bad. The reason for this bad reproducibility is that the thickness of the tris(2-aminoethyl)amine layer can not be controlled properly. Therefore the relationship between reactive particles from the plasma and the concentration of the tris(2-aminoethyl)amine on the polymer surface is not adjustable. A detailed discussion of the results is therefore not possible.

\subsection{Reduction of the Nitrile Groups}

Primary amino groups can be formed by the reduction of nitrile groups with lithium aluminium hydride [6]. This has been demonstrated for 
$\mathrm{PAN}^{3}$, but as the number of resulting surface groups depends strongly on the film preparation and the analytical method, reduction of the nitrile groups of PAN by lithium aluminum hydride was carried out to compare the results with the ammonia plasma treatment.

Annealed PAN films were put into a stirred mixture of powdered lithium aluminium hydride in dried diethylether. After the certain reaction time the polymer films were put into ethanol and washed with water. The determination of the created number of basic groups was carried out as described above. Figure 7 shows the relationship between the number of amino groups and the reaction time. The number of formed groups is in the same order of magnitude as the samples treated with the ammonia plasmas. The reaction rate is however much smaller. Even after 3 hours the equilibrium state has not been reached. This shows that the plasma chemical preparation of amino groups on the PAN surface is a faster method for practical applications. Moreover lithium aluminum hydride is difficult to handle and this method of creating amino groups is restricted to polymers containing nitrogen.

It was not possible to carry out this reaction in tetrahydrofurane as solvent, since PAN dissolves in THF. A mixture of diethylether and

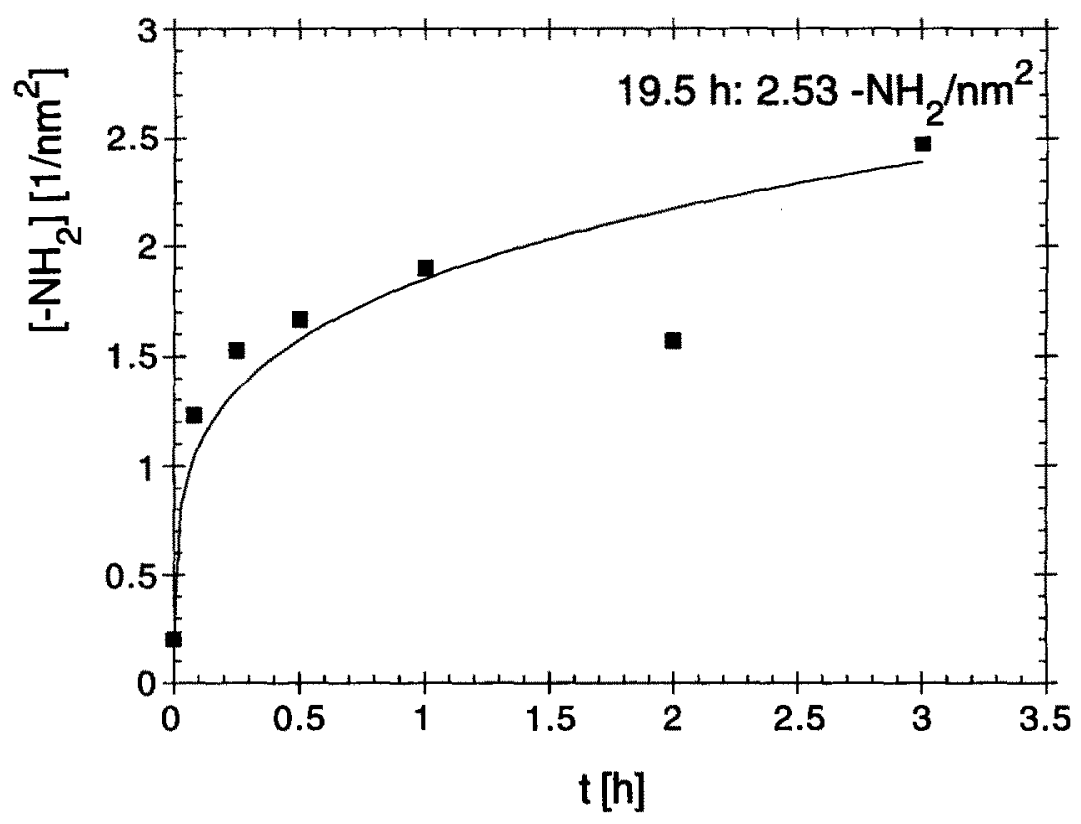

Fig. 7. Number of amino groups on the PAN surface formed by the reduction with lithium aluminium hydride as a function of the reaction time. 
tetrahydrofurane will swell the surface and may increase therefore the effective surface and the surface mobility, so that the reaction rate is raised. When the surface becomes swollen by the solvent it is however possible that lithium aluminium hydride particles become trapped in the polymer and this should be avoided.

\section{CONCLUSIONS}

The formation of amino groups on PAN surfaces is possible by the chemical reduction of the nitrile groups with lithium aluminum hydride as well as by the plasma chemical degradation of amino containing chemicals, especially of tris(2-aminoethyl)amine, on the polymer surface. The best method to obtain surface groups is the treatment with an ammonia plasma. This is not only the fastest way of amino formation, it is also the most reproducible one.

The results as described here for PAN may be applied to other polymers. One should always keep in mind that with PAN a reduction of nitrile groups can take place by the hydrogen formed in the plasma. The difference could be readily demonstrated by the comparison of PAN with PP.

Since the analysis of the surface was performed by a chemical method, only groups were detected that are available for chemical reactions in a further treatment. Additionally, this method is much faster than ESCA, and it is possible to detect amino groups independently from nitrile groups. This distinction is possible with ESCA as well but only after a further chemical modification (e.g. with trifluoro acetic acid).

\section{REFERENCES}

1 J.R. Hollahan et al., J. Appl. Polym. Sci., 13 (1969) 807.

2 P.J.C. Chappell et al., Surf. Interface Anal., 17 (1991) 143.

3 K. Matsumoto et al., U.S. patent, 4,486,549 (04/12/84).

4 E. Gurr, Synthetic Dyes, Academic Press, London, 1971, p. 202.

5 G.H. Koops, Dehydration of acetic acid by pervaporation; material science aspects, PhD. Thesis, University of Twente, 1992.

6 N.G. Gaylord, Reduction with Complex Metal Hydrides. Interscience Pub., New York, 1956, p. 731. 